\title{
Ecoeficiencia y actitudes ambientales en estudiantes de educación primaria
}

\author{
Miriam Julissa Romero Collantes \\ mromeroco88@ucvvirtual.edu.pe \\ https://orcid.org/0000-0001-8036-2631 \\ Groberti Alfredo Medina Corcuera \\ grome@ucvvirtual.edu.pe \\ https://orcid.org/0000-0003-4035-157X \\ Universidad César Vallejo \\ Chimbote- Perú
}

\section{RESUMEN}

El presente estudio tuvo como objetivo general demostrar la influencia del programa basado en ecoeficiencia para el desarrollo de las actitudes ambientales en los niños de segundo grado de la IE. N 88052 Nepeña. La investigación fue de tipo aplicada, con un enfoque cuantitativo y con un diseño cuasi experimental. La muestra fue de 48 alumnos con características similares, divididos en dos grupos de 24 estudiantes cada uno. Para recoger los datos se utilizó la técnica de la encuesta, teniendo como instrumento un cuestionario con 20 ítems sobre las cuatro dimensiones de la variable actitudes ambientales, fue validado por dos jueces expertos y para la confiabilidad se usó el Alfa de Cronbach, obteniendo $\alpha=0.778$. Los resultados muestran que el grupo experimental pasó de un pre test con un $75 \%$ de nivel malo y un $0 \%$ de nivel excelente a un post test con un 4,2\% de nivel malo y un 54,2\% de nivel excelente, además para la prueba de hipótesis se obtuvo $\mathrm{p}=0,00$, con lo cual pudimos concluir que se aceptó la hipótesis alterna, demostrándose que el programa basado en ecoeficiencia influye de manera significativa en el desarrollo de las actitudes ambientales en los niños del segundo grado.

Palabras clave: programa; ecoeficiencia; sostenibilidad; actitud ambiental; educación ambiental 


\title{
Eco-efficiency and environmental attitudes in primary school students
}

\begin{abstract}
The aimed of this research was to demonstrate the influence of the eco-efficiency-based program on the development of environmental attitudes in second grade children of E.I. $\mathrm{N}^{\circ} 88052$ Nepeña. It was an applied research, with a quantitative approach and with a quasi-experimental design. The sample consisted of 48 students with similar characteristics, divided into two groups of 24 students each. The survey technique was used to collect the data, using as an instrument a questionnaire with 20 items on the four dimensions of the variable environmental attitudes, it was validated by two expert judges and Cronbach's Alpha was used for reliability, obtaining $\alpha=0.778$. The results show that the experimental group went from a pretest with $75 \%$ of bad level and $0 \%$ of excellent level to a posttest with $4.2 \%$ of bad level and $54.2 \%$ of excellent level, in addition for the hypothesis test was obtained $\mathrm{p}=0.00$, which allowed us to conclude that the alternative hypothesis was accepted, showing that the eco-efficiency-based program significantly influences the development of environmental attitudes in students in the second grade.
\end{abstract}

Keywords: program; eco-efficiency; sustainability; environmental attitude; environmental education.

Artículo recibido: 15 noviembre. 2021 Aceptado para publicación: 10 diciembre 2021 Correspondencia: july_16_116@hotmail.com Conflictos de Interés: Ninguna que declarar 


\section{INTRODUCCIÓN}

El artículo trató de una problemática latente en todos los países, convirtiéndose en un tema preocupante a nivel mundial, la carencia de actitudes ambientales trajo consigo un gran impacto negativo en el ambiente, puesto que las formas de actuar del hombre en relación con su entorno repercutieron inmediatamente en su calidad de vida (Manisalidis, Stavropoulou, Stavropoulos y Bezirtzoglou, 2020). Por ello, en la actualidad el ambiente se encuentra muy deteriorado, debido al desmedido empleo de los recursos naturales y por una mala práctica de hábitos en favor del ambiente.

Frente a esta amenaza muchos países a nivel mundial se organizaron y realizaron acuerdos con el único fin de impedir la proliferación de algún tipo de contaminación ambiental. La falta de compromiso de estos países se vio reflejado en el Informe Mundial de la Calidad del Aire 2020, estudio que mostró las cantidades de partículas PM2.5 emitidas al ambiente. Sin duda alguna el accionar de las personas, tuvo repercusiones que provocaron y seguramente seguirán provocando daños a la Tierra. La Agencia Internacional de la Energía ya estimó un crecimiento del 130\% de las emisiones para el 2050 si se continua con las mismas actitudes frente al entorno. (IQAir AirVisual, 2020).

A raíz de este informe, se observó que el descuido hacia el ambiente conforma un nocivo problema que atañe en gran parte a toda América Latina. Según Gonzales y Steenland teniendo como fuente a los estudios realizados por la OMS, Lima es la segunda ciudad más contaminada en Latinoamérica (como se citó en Vásquez 2021), teniendo como causa principal de contaminantes a los grandes generadores de energía térmica que trabajan con diésel y gas, a las fábricas, a los parques automotores, etc.

Nepeña, lugar donde se ubica la institución no es ajena a esta realidad y a la indiferencia de sus habitantes, por tanto, observamos que las familias no poseen actitudes adecuadas para el cuidado de su entorno, estas falta se evidencian día con día dentro del colegio, observándose a la hora de entrada, recreo y salida como los estudiantes tiran la basura de los empaques de Qaliwarma o de los productos comprados en el kiosco, desperdician el agua, dejan los focos encendidos, no reciclan, pisaban las áreas verdes, etc. Esto hace pensar, que si bien es cierto el Ministerio de Educación (2017) estableció al enfoque ambiental dentro de la currícula para la formación personal de los niños, los maestros no lograron que ellos pongan en práctica lo aprendido, imposibilitando desarrollar su criticidad y poder luchar contra los problemas ambientales. Dándonos cuenta que lograr 
en los alumnos un cambio en sus estilos de vida con relación al ambiente es sumamente difícil. Por estas razones, es que se priorizó de una medida para poder trabajar con los niños y así lograr las mejoras en sus actitudes, considerando que luego ellos serán los forjadores del cambio. Por lo expuesto, es que surgió una interrogante que podría traer consecuencias positivas para el ambiente, ¿En qué medida el programa basado en ecoeficiencia influye en el desarrollo de actitudes ambientales en los niños del segundo grado de la IE. N 88052 Nepeña, 2021?

Frente a ello, podemos apreciar lo importante que fue la investigación, quedando justificada por sus implicancias prácticas, puesto que ayudó a buscar soluciones para resolver problemas ambientales que se presentaron en nuestra realidad. Considerando que fue conveniente, pues educó para el desarrollo sostenible generando cambios en las formas de pensar, en las actitudes y en las prácticas de los estudiantes, todo ello enfocado hacia el beneficio del ambiente. Tuvo una relevancia social, pues posibilitó que los niños acojan comportamientos que les ayudarán a vivir en plenitud y que por medio de ellos las futuras generaciones serán capaces de adoptar estilos de vida sin perjudicar al medio donde viven y se desarrollan. Por último, también tuvo un valor teórico, pues al término de la investigación se llegaron a generalizar los resultados, tomando al programa como una idea innovadora que otorga una visión ideal hacia el desarrollo sostenible.

En este sentido, el objetivo general de la investigación fue demostrar la influencia del programa basado en ecoeficiencia para el desarrollo de las actitudes ambientales y como objetivos específicos se tuvieron: establecer la influencia del programa basado en ecoeficiencia en las dimensiones cognitiva, afectiva, conativa y activa, así como, elaborar una propuesta de programa para desarrollar actitudes ambientales. Considerando como hipótesis: $\mathrm{H}_{1} \mathrm{El}$ programa basado en ecoeficiencia influye de manera significativa en el desarrollo de actitudes ambientales en los niños del segundo grado. Ho: El programa basado en la ecoeficiencia no influye de manera significativa en el desarrollo de actitudes ambientalistas en los niños del segundo grado.

Como esta problemática se encuentra latente a nivel mundial, existen investigaciones internacionales que se preocuparon por su estudio. Tenemos a Nihal y Ayse (2018) con su investigación titulada el efecto del programa de educación sobre la naturaleza en el nivel de conciencia ambiental en distintos niveles socioeconómicos, con los resultados obtenidos los investigadores mencionan como conclusión principal que cualquier 
programa educativo cuyas actividades estén destinadas a la interacción de la persona con la naturaleza contribuirá al desarrollo de la conciencia ambiental y por ende a la mejora del entorno sin importar su estatus socioeconómico.

En Perú, existen investigaciones como la de Alva (2019) quien estudió a la ecoeficiencia como una nueva estrategia para la educación ambiental en estudiantes de la ciudad de Tingo María, el autor menciona entre sus conclusiones que definitivamente hay una correlación importante entre la educación ambiental y la ecoeficiencia, a su vez que la ecoeficiencia se encuentra correlacionada con la sostenibilidad, las actividades cognitivas y la mejora de los comportamientos ambientales. Así mismo, Orbegoso (2019) ejecutó una investigación titulada programa de cultura ambiental en actitudes ecológicas, mencionando entre sus conclusiones que dicho programa sí influye en forma importante en las actitudes ecológicas de los educandos, esto se ve reflejado al realizar una comparación entre los datos recogidos al inicio, donde los alumnos no mostraban actitudes hacia el cuidado de su medio natural y el gran cambio que se dio luego de la aplicación del programa reflejándose en ellos el mejoramiento en sus cuatro dimensiones. Por su parte, Poma (2019) realizó una investigación titulada los hábitos de cuidado hacia la ecología y su relación con la sostenibilidad, concluyendo que existe una relación significativa entre los hábitos de conservación al ambiente y las dimensiones cognitiva, afectiva y activa de sostenibilidad, debido a que todas obtuvieron un Sig. =0,000. Razón por la cual se deduce que los hábitos y las buenas prácticas en beneficio del medio natural sí se llegan a relacionar en forma significativa con la sostenibilidad ambiental. Otra investigación fue la realizada por Trelles (2018) cuyo título fue programa mejorando mi ambiente para fomentar actitudes responsables en la protección del entorno, mencionando entre sus conclusiones, que al inicio del trabajo los dos grupos de estudios presentan actitudes poco adecuadas hacia el ambiente, pero luego de trabajar en base al programa propuesto, el grupo control no mostró ningún tipo de cambio respecto a las actitudes ecológicas, lo opuesto ocurrió con el grupo donde se aplicó el programa, notándose un progreso significativo en relación al pre - post test, esta mejora trajo consigo que los alumnos pertenecientes a este grupo tengan toda la predisposición para comprometerse a desarrollar actitudes que conlleven a una mejora en su entorno.

Nuestra región Ancash tampoco se escapa de esta grave situación, por ello existen estudios realizados para lograr combatir esta problemática. Niceforo (2020) trabajó en 
una investigación titulada capacitación de promotores para mejorar las actitudes a favor del ambiente de los alumnos. Concluyendo que, tras medir a las tres dimensiones enfocadas en el conocimiento, actitudes y lo afectivo se sabe que el grupo donde se aplicó la capacitación superó enormemente al grupo que trabajó de control, razón por la cual se deduce que la capacitación dada a los promotores mejora con una gran consideración las actitudes ambientales de los educandos.

De las investigaciones expuestas y que fueron realizadas para contribuir a la mejora del ambiente, es que se avanza a la temática de estudio. Teniendo como primera variable, al programa basado en ecoeficiencia, el cual dentro del aspecto educativo se apoya en la teoría constructivista. Uno de los aportes de gran interés para este tipo de educación centrada en el ambiente fue la teoría del aprendizaje significativo de Ausubel (1968) el cual refiere que el estudiante debe descubrir un significado de los nuevos conceptos relacionándolos con los ya aprendidos. En este tipo de aprendizajes el saber que se logra adquirir es permanente y puede ser transferido a otras circunstancias. Bajo esa línea, tenemos también a la Teoría Sociocultural de Vygotsky (1979) el cual sustentaba que todo aprendizaje se genera mediante la "zona del desarrollo próximo", a través de nuevas interacciones con personas de su entorno. Teniendo en cuenta lo dicho, el estudio se da con la interacción del niño y su entorno más cercano, con esta interacción se logra que incorpore pensamientos que contribuyan a un accionar idóneo para cuidar y defender su ambiente. Estas teorías han resultado ser muy eficaces para aumentar el conocimiento e influir en las actitudes de los estudiantes, debido a que están basadas en la teoría del constructivismo y por en donde se propone una educación para incentivar cambios de actitudes y valores, que es un fin principal de la educación centrada en la naturaleza (Noureen, Arshad, y Bashir 2020).

Otra teoría que da sustento a la primera variable es la teoría del desarrollo sostenible, la cual apareció como la integración de la dimensión ambiental dentro de los problemas globales, esta teoría se define como aquel desarrollo que permite satisfacer las necesidades de la actualidad, sin perjudicar las capacidades de las generaciones venideras, con ello se busca que los seres que habitan el planeta tengan una buena calidad de vida. (World Commission on Environment and Development, 1987).

En base a las teorías expuestas es que se creó el programa basado en ecoeficiencia el cual está conformado por una serie de actividades planificadas en donde se espera que el 
estudiante conozca, valore y desarrolle actitudes positivas que le permitan relacionarse armoniosamente con el ambiente (Schmitz y Teixeira, 2018) generando a su vez compromisos ambientales enfocados en la sostenibilidad, pues según Rosano, Rosano, Cavalcante y Marques (2020) al enfocarnos en la ecoeficiencia estamos buscando el correcto empleo de todos los recursos que provee la naturaleza sin que esto implique un alto costo en él. Esta propuesta es muy útil para el sector educativo, porque busca en la ecoeficiencia educar al estudiante fomentando la protección, conservación y recuperación del ambiente (Bofill, Cueva y Barreno, 2016). Por su parte, Al-Naqbi y Alshannag (2018) definen estas formas de propuestas como una tendencia educativa que busca que todos los actores se involucren en el proceso de aprendizaje, teniendo como objetivo causar cambios de actitudes sobre la preservación del medio ambiente, basados en los ideales que subyacen a la sostenibilidad.

En el Perú, como una manera de valorar la diversidad de recursos naturales que poseemos es que se ha venido incorporando la educación en ecoeficiencia. Por ejemplo, para el Ministerio del Ambiente (2012), este tipo de educación se aborda como una estrategia para el cambio de cultura reforzando los procesos de formación ambiental, todo ello direccionado hacia el marco del desarrollo sostenible. Por ello se afirma que una institución educativa puede ser llamada ecoeficiente cuando emplea de manera eficiente sus recursos, aminora el efecto ambiental de sus actividades e incorpora un valor agregado dentro del servicio que se pueda brindar en las escuelas: la sostenibilidad, innovación y emprendimiento social-ambiental.

El programa basado en la ecoeficiencia tiene un enfoque ambiental que plantea la relación que hay entre el entorno, la cultura y la sociedad, orientando a los procesos educativos para una formación en los estudiantes con conciencia crítica y colectiva para la protección sostenible del ambiente, así como la relación que existe con la pobreza, desigualdad social, salud y principalmente el agotamiento de los recursos naturales. (Ministerio del Ambiente, 2012; Ministerio de Educación, 2017). Con este enfoque se busca unir las áreas de aprendizaje con el fin de tratar problemáticas que acarrean no tan solo a nuestro país, sino que también a todo el mundo. Esto se puede ver reflejado en sus niveles institucionales y pedagógicos desarrollados dentro de la gestión escolar de forma transversal. 
Este programa posee cuatro dimensiones estructuradas secuencialmente. Para Matos y Pasek (2005) la planificación es el primer paso para realizar el programa, a través de ella se van a determinar los objetivos y las actividades que se ejecutarán para lograrlo, el conocimiento está referido a la pertinencia con las teorías que fundamental el programa o plan, la ejecución es la parte donde se pone en marcha lo ya planificado, por ende, es un proceso activo y por último tenemos a la evaluación momento en el cual se va a realizar el análisis de la actuación o participación en el programa.

Ejecutar este tipo de investigación involucra un cambio total de actitudes ambientales por parte de los educandos. Esto significa que cambiarán la respuesta o el comportamiento que tienen frente a problemas que ocurren en el ambiente, estas formas de respuestas se darán teniendo en cuenta los valores y creencias del individuo al momento de juzgar algunos aspectos de la realidad, beneficiando o perjudicando al medio que nos rodea. (Barja et al. 2019). Frente a ello, Côrtes, Dias, Da Silva y Vieira (2016) refieren que los seres humanos solo realizan actitudes responsables cuando son conocedores de la problemática ambiental, lo que los motiva para generar cambios cualitativos.

Frente a lo dicho existen teorías que fundamentan esta variable, una de ellas es la Teoría Moral de Kohlberg (1981), el cual considera que la integralidad del individuo desde el plano moral debe orientarse en todas sus dimensiones, para él la moralidad se relaciona con los avances cognitivos y conductuales, teniendo como fin la obtención de comportamientos morales que permitan un adecuado actuar, acordes con valores aceptados libre y responsablemente en pro de la mejora ambiental. Para reforzar lo mencionando, Sánchez y Balaguera (2012) manifiesta que la educación ambiental es la encargada de los aspectos prácticos y cognitivos, pero que no genera ningún tipo de resultados en los cambios de las actitudes, conductas o valores sustentables. Novo (1995) reafirma mencionado que para este tipo de educación la cuestión del conocimiento es importante pero insuficiente. Otra teoría que da sustento a la actitud ambiental es la Teoría Ecológica de Bronfenbrenner (1987), concede entender cómo es que actúa el medio natural en el crecimiento de los seres humanos, interviniendo en su desarrollo integral. Para el autor su teoría comprende al ambiente para el crecimiento personal, influido por las costumbres, leyes y su entorno. Por su parte Gardner (1995) puntualiza el amor y la cercanía que tiene el hombre para con su entorno, considerando que a medida que el niño 
va creciendo, crea su inteligencia convirtiéndolo en una excelente persona cuyas capacidades pueden ser aprovechada para defender su entorno natural.

Conjuntamente a estas teorías, Corraliza, Martín, Moreno y Berenguer (2004), establecen cuatro dimensiones para esta variable a investigar. La dimensión cognitiva, hace alusión al grado de investigación, así como también a la formación en temas referentes a los problemas que puedan existir en el medio. Esto debido a que se hace imprescindible poseer los conocimientos esenciales con lo cual se pueda comprender dichos problemas y con ellos tener la capacidad de proponer soluciones ideales, la segunda dimensión que incumbe a las emociones es la afectiva, Marcinkowski y Reid (2019) indican que la susceptibilidad hacia los temas del ambiente permite que los seres humanos tengan una posición de amor y respeto a su medio natural. En consecuencia, trabajarla implicará poner en práctica los valores a favor del ambiente. Por su parte la tercera dimensión conativa, según Corraliza, et al. (2004) refieren que tiene su enfoque en rechazar o preferir hechos ambientales, que permite tomar determinaciones que involucran valorar motivos y actitudes que facilitan actuar de forma asertiva con su entorno. La cuarta dimensión es la activa, para Jiménez y Lafuente (2010) alude al actuar que toma el ser humano ante las múltiples situaciones que se puedan presentar en relación con el cuidado de su medio.

\section{ESTRATEGIAS METODOLÓGICAS O MATERIALES Y MÉTODOS}

El estudio fue de tipo aplicada, la cual está orientada a resolver un problema que aqueja nuestra realidad. El enfoque que se utilizó fue cuantitativo, con un método hipotéticodeductivo. Se empleó un diseño cuasi experimental, cuyo diagrama correspondiente es:

\section{Donde:}

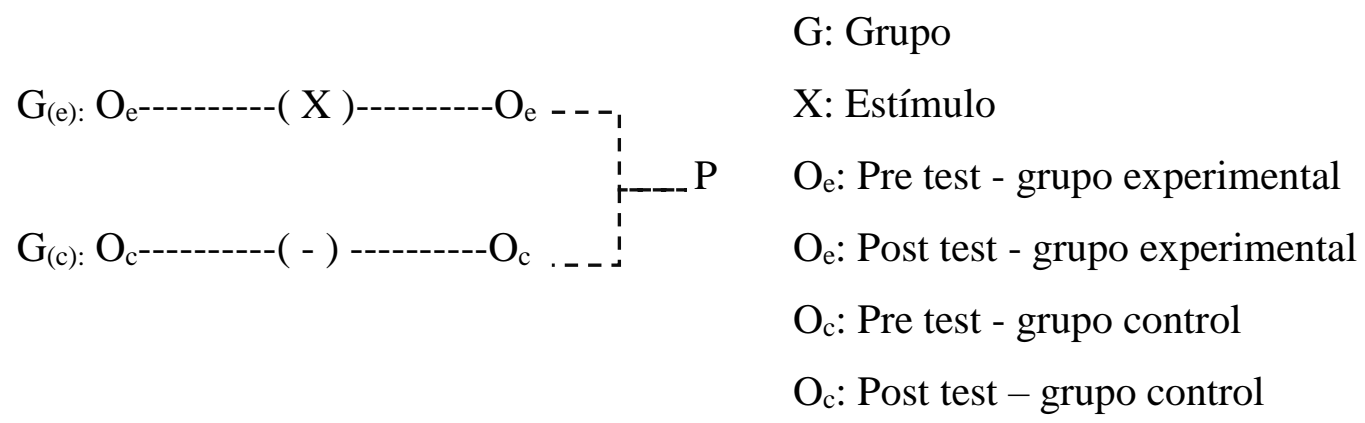

La investigación tuvo una población conformada pr P: Propuesta

Evaristo Villacré, con una muestra de 48 alumnos del segundo grado de primaria repartidos equitativamente entre el grupo control y experimental. Se empleó la técnica de la encuesta, teniendo como instrumento de evaluación al cuestionario Pre test -Post test 
sobre actitudes ambientales, el cual contó con 20 ítems distribuidos entre las cuatro dimensiones de la variable dependiente. La calificación del cuestionario fue de cero a veinte, cada ítem bien contestado sumó un punto, con los puntajes establecidos se pudo obtener valores sobre las actitudes ambientales de los alumnos, quedando establecido de la siguiente manera: mala actitud ambiental (de 0 a 10 puntos), regular actitud ambiental (11 a 14 puntos) buena actitud ambiental (de 15 a 17 puntos) y excelente actitud ambiental (de 18 a 20 puntos). Dicho instrumento fue validado por dos expertos en el área, para la confiabilidad se trabajó con una evaluación piloto contando con la participación de 15 estudiantes, se hizo uso del Alfa de Cronbach, obteniendo un resultado de $\alpha=0.778$. Para procesar la información se utilizó la base de datos Excel 2019 y SPSS 25, empleando el análisis de la estadística descriptiva el cual permitió ordenar datos en tablas. Para la contrastación de hipótesis se usó de la estadística inferencial utilizando la prueba de normalidad Shapiro - Wilk y la U de Mann- Whitney.

\section{RESULTADOS Y DISCUSIÓN}

\subsection{Resultados}

\section{Análisis descriptivo}

Tabla 1 Resultado del pre y post test del grupo control y experimental en el desarrollo de las actitudes ambientales

\begin{tabular}{|c|c|c|c|c|c|c|c|c|}
\hline \multirow{3}{*}{ Nivel } & \multicolumn{4}{|c|}{ Control } & \multicolumn{4}{|c|}{ Experimental } \\
\hline & \multicolumn{2}{|c|}{ Pre } & \multicolumn{2}{|c|}{ Post } & \multicolumn{2}{|c|}{ Pre } & \multicolumn{2}{|c|}{ Post } \\
\hline & $\mathbf{F i}$ & $\%$ & $\mathbf{F i}$ & $\%$ & fi & $\%$ & fi & $\%$ \\
\hline Malo & 17 & $70,8 \%$ & 18 & $75,0 \%$ & 18 & $75,0 \%$ & 1 & $4,2 \%$ \\
\hline Regular & 4 & $16,7 \%$ & 3 & $12,5 \%$ & 2 & $8,3 \%$ & 3 & $12,5 \%$ \\
\hline Bueno & 3 & $12,5 \%$ & 3 & $12,5 \%$ & 4 & $16,7 \%$ & 7 & $29,2 \%$ \\
\hline Excelente & 0 & $0,0 \%$ & 0 & $0,0 \%$ & 0 & $0,0 \%$ & 13 & $54,1 \%$ \\
\hline Total & 24 & $100 \%$ & 24 & $100,0 \%$ & 24 & $100,0 \%$ & 24 & $100 \%$ \\
\hline
\end{tabular}

Fuente: Base de datos del Pre y Post Test del grupo experimental y control

\section{Interpretación:}

En la tabla 1 respecto al pre test, el grupo control y experimental se encontraban con un mayor porcentaje en el nivel malo con un $70.8 \%$ y $75 \%$, ninguno de los grupos pudo ubicarse en el nivel excelente, luego de aplicar el programa, se mostró que el grupo control no había tenido cambios significativos, caso contrario fue lo ocurrido con el grupo experimental, pues se constató que solo el 4,2\% de alumnos se encontraron en un nivel 
malo, teniendo el mayor porcentaje representado por el $54,1 \%$ de alumnos que lograron exitosamente ubicarse en el nivel excelente, seguidos del 29,2\% en el nivel bueno.

\section{Tabla 2}

Resultado de la dimensión cognitiva en el desarrollo de las actitudes ambientales

\begin{tabular}{|c|c|c|c|c|c|c|c|c|}
\hline \multirow{3}{*}{ Nivel } & \multicolumn{4}{|c|}{ Control } & \multicolumn{4}{|c|}{ Experimental } \\
\hline & \multicolumn{2}{|c|}{ Pre } & \multicolumn{2}{|c|}{ Post } & \multicolumn{2}{|c|}{ Pre } & \multicolumn{2}{|c|}{ Post } \\
\hline & fi & $\%$ & $\mathbf{f i}$ & $\%$ & fi & $\%$ & $\mathbf{F i}$ & $\%$ \\
\hline Malo & 14 & $58,3 \%$ & 15 & $62,5 \%$ & 15 & $62,5 \%$ & 1 & $4,2 \%$ \\
\hline Regular & 6 & $25,0 \%$ & 5 & $20,8 \%$ & 4 & $16,7 \%$ & 2 & $8,3 \%$ \\
\hline Bueno & 4 & $16,7 \%$ & 4 & $16,7 \%$ & 5 & $20,8 \%$ & 7 & $29,2 \%$ \\
\hline Excelente & 0 & $0,0 \%$ & 0 & $0,0 \%$ & 0 & $0,0 \%$ & 14 & $58,3 \%$ \\
\hline Total & 24 & $100,0 \%$ & 24 & $100,0 \%$ & 24 & $100,0 \%$ & 24 & $100,0 \%$ \\
\hline
\end{tabular}

Fuente: Base de datos del Pre y Post Test del grupo experimental y control

\section{Interpretación:}

En la tabla 2, se halló que antes de la experiencia para esta dimensión los niños de ambos grupos tenían mayor preponderancia en un nivel malo con un 58,3\% para el grupo control y un $62,5 \%$ para el experimental, ninguno de los grupos obtuvo un porcentaje para el nivel excelente, llegando solo al nivel bueno con un 16,7\% para ambas muestras. Tras la aplicación del programa se pudo observar que el grupo control no presentó cambios importantes, a diferencia del grupo experimental, que logró un mayor porcentaje en el nivel excelente con una 58,3\% y solo el $4.2 \%$ siguió ubicándose en el nivel malo.

\section{Tabla 3}

Resultado de la dimensión afectiva en el desarrollo de las actitudes ambientales

\begin{tabular}{|c|c|c|c|c|c|c|c|c|}
\hline \multirow{3}{*}{ Nivel } & \multicolumn{4}{|c|}{ Control } & \multicolumn{4}{|c|}{ Experimental } \\
\hline & \multicolumn{2}{|c|}{ Pre } & \multicolumn{2}{|c|}{ Post } & \multicolumn{2}{|c|}{ Pre } & \multicolumn{2}{|c|}{ Post } \\
\hline & fi & $\%$ & fi & $\%$ & fi & $\%$ & fi & $\%$ \\
\hline Malo & 10 & $41,7 \%$ & 8 & $33,3 \%$ & 12 & $50,0 \%$ & 0 & $0,0 \%$ \\
\hline Regular & 8 & $33,3 \%$ & 11 & $45,9 \%$ & 6 & $25,0 \%$ & 1 & $4,2 \%$ \\
\hline Bueno & 6 & $25,0 \%$ & 5 & $20,8 \%$ & 6 & $25,0 \%$ & 7 & $29,2 \%$ \\
\hline
\end{tabular}




\begin{tabular}{|c|c|c|c|c|c|c|c|c|}
\hline Excelente & 0 & $0,0 \%$ & 0 & $0,0 \%$ & 0 & $0,0 \%$ & 16 & $66,6 \%$ \\
\hline Total & 24 & $100,0 \%$ & 24 & $100,0 \%$ & 24 & $100,0 \%$ & 24 & $100,0 \%$ \\
\hline
\end{tabular}

Fuente: Base de datos del Pre y Post Test del grupo experimental y control

\section{Interpretación:}

En la tabla 3 se pudo apreciar que antes de aplicar el programa el 41,7 \% del grupo control y el 50,0\% del grupo experimental se ubicaron en el nivel malo, ambos grupos no obtuvieron ningún porcentaje en el nivel excelente, llegando con un $25 \%$ tanto para el grupo control como experimental a ocupar el nivel bueno, luego de aplicar la experiencia se notó que el grupo control mantenía similares resultados al pre test, caso distinto al grupo experimental, que logró que ningún educando se ubique en el nivel malo y que la mayoría de sus alumnos representado por un $66,6 \%$ se encuentren en un nivel excelente.

\section{Tabla 4}

Resultado de la dimensión conativa en el desarrollo de las actitudes ambientales

\begin{tabular}{|l|c|c|c|c|c|c|c|c|}
\hline \multirow{2}{*}{ Nivel } & \multicolumn{4}{|c}{ Pre } & \multicolumn{2}{c}{ Post } & \multicolumn{3}{c|}{ Experimental } \\
& $\mathbf{f i}$ & $\mathbf{\%}$ & Total & $\mathbf{\%}$ & Total & \% & Total & \% \\
\hline Malo & 13 & $54,1 \%$ & 11 & $45,9 \%$ & 14 & $58,3 \%$ & 0 & $0,0 \%$ \\
\hline Regular & 7 & $29,2 \%$ & 8 & $33,3 \%$ & 6 & $25,0 \%$ & 1 & $4,2 \%$ \\
\hline Bueno & 4 & $16,7 \%$ & 5 & $20,8 \%$ & 4 & $16,7 \%$ & 10 & $41,7 \%$ \\
\hline Excelente & 0 & $0,0 \%$ & 0 & $0,0 \%$ & 0 & $0,0 \%$ & 13 & $54,1 \%$ \\
\hline Total & $\mathbf{2 4}$ & $\mathbf{1 0 0 , 0} \%$ & $\mathbf{2 4}$ & $\mathbf{1 0 0 , 0} \%$ & $\mathbf{2 4}$ & $\mathbf{1 0 0 , 0} \%$ & $\mathbf{2 4}$ & $\mathbf{1 0 0 , 0 \%}$ \\
\hline
\end{tabular}

Fuente: Base de datos del Pre y Post Test del grupo experimental y control

\section{Interpretación:}

En la tabla 4, se halló que antes de aplicar la experiencia el grupo control y experimental obtuvieron un $45,9 \%$ y $58,3 \%$ de estudiantes en el nivel malo, el $16,7 \%$ y el $20,8 \%$ lograron ubicarse en el nivel bueno, sin obtener ningún porcentaje en el nivel excelente. Después de la experiencia el grupo control no obtuvo ningún cambio significativo a diferencia del grupo experimental que no reportó a ningún estudiante en el nivel malo, mientras que el $54,1 \%$ se ubicaron en el nivel excelente y el $41,7 \%$ en el nivel bueno.

\section{Tabla 5}

Resultado de la dimensión activa en el desarrollo de las actitudes ambientales

\begin{tabular}{|c|c|c|c|c|c|c|c|}
\hline \multirow{3}{*}{ Nivel } & \multicolumn{4}{|c|}{ Control } & \multicolumn{2}{|c|}{ Experimental } & \\
\hline & & & & & & & \\
\hline & fi & $\%$ & fi & $\%$ & fi & $\%$ & f \\
\hline
\end{tabular}




\begin{tabular}{|l|c|c|c|c|c|c|c|c|}
\hline Malo & 12 & $50,0 \%$ & 13 & $54,1 \%$ & 13 & $54,1 \%$ & 0 & $0,0 \%$ \\
\hline Regular & 9 & $37,5 \%$ & 7 & $29,2 \%$ & 6 & $25,0 \%$ & 2 & $8,4 \%$ \\
\hline Bueno & 3 & $12,5 \%$ & 4 & $16,7 \%$ & 5 & $20,9 \%$ & 8 & $33,3 \%$ \\
\hline Excelente & 0 & $0,0 \%$ & 0 & $0,0 \%$ & 0 & $0,0 \%$ & 14 & $58,3 \%$ \\
\hline Total & $\mathbf{2 4}$ & $\mathbf{1 0 0 , 0 \%}$ & $\mathbf{2 4}$ & $\mathbf{1 0 0 , 0} \%$ & $\mathbf{2 4}$ & $\mathbf{1 0 0 , 0} \%$ & $\mathbf{2 4}$ & $\mathbf{1 0 0 , 0} \%$ \\
\hline
\end{tabular}

Fuente: Base de datos del Pre y Post Test del grupo experimental y control

\section{Interpretación:}

En la tabla 5 se pudo apreciar que antes de la experiencia el grupo control y experimental obtuvieron el 50,0\% y el 54,1\% de nivel malo, no hubo porcentajes de ningún grupo para el nivel excelente, llegando solo con un $12,5 \%$ y 16,7\% al nivel bueno. Luego de aplicar el programa el grupo control mostró resultados parecidos al del pre test, en cambio el grupo experimental evidenció cambios muy significativos, logrando tener al 58,3\% en el nivel excelente, al 33,3\% en el nivel bueno y $0 \%$ en el nivel malo.

\section{Análisis Inferencial}

\section{Tabla 6}

Resultado de la prueba de hipótesis del post test de las actitudes ambientales del grupo control y grupo experimental

\begin{tabular}{l|c} 
& AA_POST_GC_GEX \\
\hline U de Mann-Whitney & 20,500 \\
\hline W de Wilcoxon & 320,500 \\
\hline Z & $-5,556$ \\
\hline Sig. asintótica (bilateral) &, 000 \\
$\quad$ Fuente: Data de Post Test del grupo control y experimental & \\
Interpretación: &
\end{tabular}

Para realizar la contrastación de hipótesis se hizo uso de prueba de normalidad Shapiro Wilk debido a que muestra fue menor a 50 alumnos y la U de Mann- Whitney por ser una muestra con datos no paramétricos.

En la tabla 6 se muestran los resultados obtenidos de la prueba de hipótesis, evidenciándose resultados óptimos tras la aplicación del programa, puesto que en su significancia los resultados arrojaron $\mathrm{p}=0,00<0,05$ para la variable actitud ambiental.

\subsection{Discusión}

El estudio tuvo como fin principal demostrar la influencia del programa basado en ecoeficiencia para el desarrollo de las actitudes ambientales. En la tabla 1, se aprecia los 
resultados obtenidos de dicho objetivo, evidenciándose que solo el grupo experimental tuvo un cambio significativo después de la experiencia. Estos resultados coinciden con los encontrados por Orbegoso (2019) y Trelles (2018) quienes refieren que luego de la aplicación de sus programas los resultados indicaron un incremento en las actitudes en beneficio del ambiente de sus estudiantes. Si bien es cierto que los resultados fueron parecidos, debemos de tener en cuenta que estos investigadores centraron su estudio en un programa para fomentar la cultura ambiental y mejorar el entorno, lo que la diferencia de esta investigación que está centrada en la ecoeficiencia, permitiendo según Rosano et al. (2020) y World Commission on Environment and Development, (1987) generar en los estudiantes compromisos ambientales enfocados en la sostenibilidad, dando un correcto uso de los recursos naturales para lograr satisfacer nuestras necesidades sin que perjudique las capacidades de las próximas generaciones. Frente a ello, el Ministerio del Ambiente (2012) acota que una institución educativa se convierte en ecoeficiente cuando se emplea de manera eficiente los recursos de la naturaleza, aminorando los efectos ambientales de sus actividades e incorporando el valor agregado de la sostenibilidad, innovación y emprendimiento social-ambiental en las escuelas.

En la tabla 2 se puede apreciar los resultados del primer objetivo específico referido a la dimensión cognitiva de las actitudes ambientales, tras la aplicación del programa se pudo observar que el grupo control no presentó cambios importantes, diferente fue lo ocurrido con el grupo experimental, demostrándose que para esta dimensión existieron mejoras significativas tras el programa. Frente a ello, Côrtes et al. (2016) manifiestan que los seres humanos solo realizan actitudes responsables cuando son conocedores de la problemática ambiental, lo que los motiva para generar cambios cualitativos, idea que es asumida también por Corraliza et al. (2004) manifestando que esta dimensión se ocupa de la formación en temas referentes a los problemas que puedan existir en el medio, haciéndose vital poseer los conocimientos esenciales con lo cual se pueda comprender dichos problemas y con ellos tener la capacidad de proponer soluciones ideales. Novo (1995) refuta a estos dos autores, pues afirma que lo cognitivo es muy valioso pero que trabajando solo con conocimiento será escaso el desarrollo de actitudes ambientales.

En la tabla 3 se puede apreciar los resultados del segundo objetivo específico referido a la dimensión afectiva de las actitudes ambientales, luego de aplicar la experiencia se notó que el grupo control mantenía similares resultados al pre test, caso distinto al grupo 
experimental, que logró que ningún estudiante se ubique en el nivel malo. Igual resultado obtuvo Niceforo (2020) quien menciona que tras medir a la dimensión afectiva se supo que el grupo donde se aplicó la capacitación superó enormemente al grupo control. Marcinkowski y Reid (2019) indican que la susceptibilidad hacia los temas del ambiente permite que los seres humanos tengan una posición de amor y respeto a su medio natural, por ello trabajar esta dimensión dentro del programa permite poner en práctica los valores a favor del ambiente, reafirmando así la teoría de Kohlberg (1981) teniendo como fin la obtención de comportamientos morales que permitan un adecuado actuar, acordes con valores aceptados libre y responsablemente en pro de la mejora ambiental.

En la tabla 4 se puede apreciar los resultados del tercer objetivo específico referido a la dimensión conativa de las actitudes ambientales, tras la aplicación del programa el grupo control no presentó cambios importantes, a diferencia del grupo experimental que no reportó a ningún estudiante en el nivel malo, ocupando mayores porcentajes en el nivel excelente. Orbegoso (2019) obtuvo similares resultados al concluir su programa, al realizar las comparaciones del antes y después, el grupo experimental evidenció el mejoramiento de la dimensión conativa, lo que permitió que los estudiantes puedan desarrollar actitudes a favor del ambiente. Corraliza, et al. (2004) manifiesta que esta dimensión tiene su enfoque en rechazar o preferir hechos ambientales, que permite tomar decisiones que involucran valorar motivos y actitudes que facilitan actuar de forma asertiva y ética con su entorno. Razón por la cual Barja, et al. (2019) manifiesta que los comportamientos se darán teniendo en cuenta los valores y creencias del individuo al momento de juzgar algunos aspectos de la realidad.

En la tabla 5 se puede apreciar los resultados del cuarto objetivo específico referido a la dimensión activa de las actitudes ambientales. Luego de aplicar el programa, el grupo control mostró resultados parecidos al del pre test, en cambio el grupo experimental evidenció cambios muy significativos, evidenciando la influencia del programa en dicha dimensión. Otras investigaciones como la de Poma (2019) estableció una relación importante entre los hábitos de conservación al ambiente y la dimensión activa de sostenibilidad. Jiménez y Lafuente (2010) manifiestan que esta dimensión está guiada a lograr una actuar coherente en el ser humano, participando de forma ordenada en todas las acciones que busquen el progreso de la interrelación con el entorno. 
En la tabla 6 se muestran los resultados obtenidos en la prueba de hipótesis del post test de los dos grupos, evidenciándose resultados óptimos tras la aplicación del programa, puesto que en su significancia los resultados arrojaron $\mathrm{p}=0,00<0,05$ para la variable actitud ambiental, razón por la cual se aceptó la hipótesis alterna, demostrándose que el programa basado en ecoeficiencia influye de manera significativa en el desarrollo de las actitudes ambientales en los niños del segundo grado. Similares resultados se obtuvieron con Poma (2019) al finalizar su estudio obtuvo un Sig.=0 ,000 con lo cual se concluyó que hay una relación muy importante entre las variables hábitos de cuidado hacia la ecología y las dimensiones cognitiva, afectiva y activa de sostenibilidad. Nihal y Ayse (2018) mencionan como conclusión principal de su trabajo, que todo programa educativo cuyas actividades estén destinadas a la interacción de la persona con la naturaleza contribuirá a la mejora y favorecimiento del medio natural.

El programa basado en la ecoeficiencia también sigue esa línea y se sustenta en teorías como las de Vygotsky (1979) el cual menciona que el aprendizaje se da por medio de las interacciones del estudiante con su entorno y la de Bronfenbrenner (1987) quien manifiesta que el medio ambiente actúa para el crecimiento de las personas, interviniendo de esa manera en su desarrollo integral, teniendo en cuenta cada una de las dimensiones trabajadas. Por todo lo expuesto, vemos la relevancia del programa, el cual se centró en la ecoeficiencia para desarrollar actitudes ambientales en los estudiantes, convirtiéndose como la manifiesta Bofill, et al. (2016) en una propuesta útil para el sector educativo porque busca en la ecoeficiencia educar al estudiante fomentando la protección, conservación y recuperación del ambiente. Por su parte, Al-Naqbi y Alshannag (2018) definen estas formas de propuestas como una tendencia educativa que busca que todos los actores se involucren en el proceso de aprendizaje, teniendo como objetivo causar cambios de actitudes, basados en los ideales que subyacen a la sostenibilidad.

\section{CONCLUSIONES}

- Se concluyó que el programa basado en ecoeficiencia influye significativamente en el desarrollo de actitudes ambientales en los niños del segundo grado; el grupo experimental desarrolló actitudes para proteger al ambiente, tal como se evidencia en los resultados obtenidos en la tabla 6 donde el Sig. de la variable dependiente fue de 0,00. Aceptándose la hipótesis alterna. 
- Se concluyó que el programa basado en ecoeficiencia influye significativamente en la dimensión cognitiva de las actitudes ambientales en los niños del segundo grado; en base a los resultados obtenidos de la tabla 2, se afirma que el grupo experimental logró desarrollar la dimensión cognitiva, consolidando su capacidad de investigación, así como sus conocimientos sobre temas ambientales.

- Se concluyó que el programa basado en ecoeficiencia influye en la dimensión afectiva de las actitudes ambientales en los niños de segundo grado; a partir de los resultados de la tabla 3, se afirma que el grupo experimental asumió una posición de amor y respeto a su medio natural, asumiendo un compromiso de preservación ambiental.

- Se concluyó que el programa basado en ecoeficiencia influye en la dimensión conativa de las actitudes ambientales en los niños de segundo grado; en función a los resultados de la tabla 4, se demuestra que el grupo experimental adquirió mayor interés por participar en actividades que incentiven el cuidado ambiental, siendo capaces de dar propuestas y de formar parte de la solución a la problemática existente.

- Se concluyó que el programa basado en ecoeficiencia influye en la dimensión activa de las actitudes ambientales en los niños de segundo grado; en base a los resultados de la tabla 5, se afirma que el grupo experimental logró una destacada mejora, al evidenciar que han desarrollado sus actitudes, demostrando el gran interés por ser partícipes activos de la lucha en beneficio del ambiente.

- Se elaboró una propuesta de programa basado en ecoeficiencia para desarrollar actitudes ambientales, con el fin de convertirse en un modelo a seguir, para que los maestros puedan incorporar los términos de ecoeficiencia y sostenibilidad en la vida diaria de los educandos.

\section{LISTA DE REFERENCIAS}

Al-Naqbi, A., y Alshannag, Q. (2018). The status of education for sustainable development and sustainability knowledge, attitudes, and behaviors of UAE University students. International Journal of Sustainability in Higher Education, 19(3), 566-588. doi: 10.1108/IJSHE-06-2017-0091

Alva, W. (2019). Ecoeficiencia: Nueva estrategia para la educación ambiental en instituciones educativas. Investigación Valdizana, 13(2), 77-84. doi: https://doi. org/10.33554/riv.13.2.233 
Ausubel, D. (1968). Educational psychology: a cognitive view. New York: Holt, Rinehart and Winston.

Barja, J., Otoya, O., Vega, E., Moreno, N., Loli, A. (2019). Attitudes towards the investigation of rotating obstetrics inmates in a Lima-Peru hospital. Faculty of Human Medicine URP 19(4),53-59. doi: 10.25176/RFMH.v19i4.2341

Bofill, A., Cueva, L., y Barreno, D. (2016). Propuesta de un programa de gestión ambiental para la Universidad Metropolitana, sede Machala. Revista Universidad y Sociedad, 8(3), 23-30. Recuperado de http://scielo.sld.cu/scielo.php?script=sci_ arttext\&pid= S2218-3620201600030 0003

Bronfenbrenner, U. (1987). La ecología del desarrollo humano. Barcelona, España: Paidós.

Corraliza, J., Martín, R., Moreno, M., y Berenguer, J. (2004). La investigación de la conciencia ambiental. Un enfoque psicosocial. Revista Persona Sociedad y Medio Ambiente, 106-120.

Côrtes, P., Dias, A., Da Silva, M., y Vieira, J. (2016). Environmental behavior: a comparative study between brazilian and portuguese students. Ambiente \& Sociedade, 19(3), 113-134. doi: https://dx.doi.org/10.1590/1809-4422ASOC 13909 9V1932016

Gardner, H. (1995). Inteligencias Múltiples. La teoría en la práctica. Barcelona, España: Paidós.

IQAir AirVisual. (2020). World's most polluted cities 2020 (PM2.5). Recuperado de https://www.iqair.com/world-most-polluted-cities

Jiménez, M., y Lafuente, R. (2010). Definición y medición de la conciencia ambiental. Revista Internacional De Sociología, 68(3), 731-755. doi: https://doi.org/10.3989/ris.2008. 11.03

Kohlberg, L. (1981). The Philosophy of Moral Development Moral Stages and the Idea of Justice. San Francisco: CA: Harper\&Row Pubs.

Manisalidis, I., Stavropoulou, E., Stavropoulos, A., y Bezirtzoglou, E. (2020). Environmental and Health Impacts of Air Pollution: A Review. Front. Public Health 8:14. doi: 10.3389/fpubh.2020.00014

Marcinkowski,T., y Reid, A. (2019) Reviews of research on the attitude-behavior relationship and their implications for future environmental 
education research. Environmental Education Research, 25(4), 459-471, doi: $10.1080 / 13504622.2019 .1634237$

Matos, Y., y Pasek, E (2005). Planificación y ejecución de la investigación en equipo: un constructo. Revista de Artes y Humanidades UNICA, 6(14),102-122.Recuperado de https://www.redalyc.org/articulo.oa? $\mathrm{id}=170118766006$

Ministerio del Ambiente. (2012). Ciudadanía Ambiental: Guía Educación en Ecoeficiencia.Perú.

Ministerio de Educación. (2017). Currículo Nacional. Perú.

Niceforo, T. (2020). Capacitación de Promotores para las Actitudes Ambientales en las Instituciones Educativas Públicas Rurales Unidocentes del Distrito de La Libertad, Provincia Huaraz Región Ancash. (Tesis doctoral). Universidad Nacional de Educación Enrique Guzmán y Valle, Lima, Perú.

Nihal, Y. y Ayse, T. (2018). The Effect of Nature Education Program on the Level of Environmental Awareness of the Elementary School Students from Different Socioeconomic Status. Universal Journal of Educational Research, 6(9), 19281937. doi: 10.13189/ujer.2018.060911

Noureen, G., Arshad, T., y Bashir, M. (2020). Effect of Constructivist Teaching Approach on Student's Achievement in Science at Elementary Level. Pakistan Social Sciences Review, 4(3), 904-911. doi: 10.35484/pssr.2020(4-III)64

Novo, M. (1995), La educación ambiental. Bases éticas, conceptuales metodológicas. Madrid: Editorial Universitas,

Orbegoso, M. (2019) Programa de cultura ambiental en actitudes ecológicas de los estudiantes del tercer grado de educación secundaria, Angasmarca-2017. SCIÉNDO, 22(1), 23-30. doi: https://doi.org/10.17268/sciendo.2019.003

Poma, S. (2019). Los hábitos de conservación del medio ambiente y su relación con la sostenibilidad ambiental de la Institución Educativa $N^{\circ} 20265$ “Los Atavillos” de la Perla, Chaupis -Huaral-2016. (Tesis doctoral). Universidad Nacional de Educación Enrique Guzmán y Valle, Lima, Perú.

Rosano, C., Rosano, C., Cavalcante, E., y Marques, A. (2020). Spatial Dependency of Eco-Efficiency of Agriculture in São Paulo. BBR. Brazilian Business Review, 17(3), 328-343.doi: https://doi.org/10.15728/bbr.2020.17.3.5 
Sánchez, H. y Balaguera, C. (2012). Conciencia moral ambiental: pertinencia de una mediación escolar. Rastros Rostros, 14(28), 129-136.

Schmitz, G., Teixeira, J. (2018). Environmental Education Program as a Tool to Improve Children's Environmental Attitudes and Knowledge. Education, 8(2), 15-20. doi: 10.5923/j.edu.20180802.01

Trelles, I. (2018). Programa “Mejorando mi entorno” para desarrollar comportamientos ecológicos responsables en el cuidado del medio ambiente en las estudiantes de 3er año de Educación Secundaria de la Institución Educativa Nuestra señora de Fátima. Piura 2018. (Tesis doctoral). Universidad César Vallejo, Ica, Perú.

Vásquez, V., Parras, E., Tapia, V., Paz, V., Rojas, J., Sánchez, O., y Gonzales, G. (2021). Association between air pollution in Lima and the high incidence of COVID-19: findings from a post hoc analysis. BMC Public Health 21, 1161. doi: https://doi.org/10.1186/s12889-021-11232-7

Vigotsky, L. (1979). Pensamiento y Lenguaje. Buenos Aires: Latauro.

World Commission on Environment and Development (1987). Our common future. The Brundtland Report. Oxford: Oxford University Press. 